\title{
Correlations in back-to-back hadron production in SIDIS
}

\author{
Harut Avakian* \\ Jefferson Lab \\ E-mail: avakian@jlab.org
}

\section{Silvia Pisano}

$L N F-I N F N$

E-mail: pisanoselnf.infn.it

The Deep Inelastic Scattering (DIS) proved to be a great tool in testing of the theory of strong interactions. Semi-Inclusive DIS (SIDIS), with detection of an additional hadron allowed first studies of 3D structure of the nucleon, moving the main focus from testing the QCD to understanding of strong interactions and quark gluon dynamics to address a number of puzzles accumulated in recent years. Detection of two hadrons in SIDIS, which is even more complicated, provides access to details of quark gluon interactions inaccessible in single-hadron SIDIS, providing a new avenue to study the complex nucleon structure. Large acceptance of the CLAS detector at Jefferson Lab, allowing detection of two hadrons, produced back-to-back (b2b) in the current and target fragmentation regions, provides a unique possibility to study the nucleon structure in target fragmentation region, and correlations of target and current fragmentation regions.

XXIV International Workshop on Deep-Inelastic Scattering and Related Subjects

11-15 April, 2016

DESY Hamburg, Germany

\footnotetext{
* Speaker.
} 
The quark-gluon dynamics manifests itself in a set of non-perturbative functions describing all possible spin-spin and spin-orbit correlations. Many experiments worldwide are currently trying to pin down various effects related to the nucleon structure through semi-inclusive deep-inelastic scattering (HERMES at DESY [1, 2, 3, 4], COMPASS at CERN [5], Jefferson Lab [6, 7, 8, 9]) polarized proton-proton collisions (PHENIX, STAR and BRAHMS at RHIC) [10, 11, 12], and electron-positron annihilation (Belle and BaBar) [13, 14, 15, 16].

Azimuthal distributions of final state particles in hard scattering processes, in particular, are sensitive to the orbital motion of quarks and play an important role in the study of transverse momentum distributions (TMDs) of quarks in the nucleon. Transverse momentum dependent distributions of partons, encoded in TMDs have been widely recognized as key objectives of the JLab $12 \mathrm{GeV}$ upgrade and a driving force behind construction of the Electron Ion Collider (EIC). Correlations of the spin of the target or/and the momentum and the spin of quarks, combined with final state interactions define the azimuthal distributions of produced particles.

Combination of measurements in a large $Q^{2}$ range from HERMES, COMPASS and JLab, extended to EIC would allow studies of evolution effects and control possible higher twist contributions in the measurements of TMD observables in general, and of the Sivers asymmetry in particular. Wide acceptance of CLAS12 detector at Jefferson Lab (JLab) and EIC would allow also checks of Sivers effect in the target fragmentation region, where it is expected to change the sign [17]. Much higher $Q^{2}$ range accessible at JLab12 with CLAS12 and EIC would allow for studies of $Q^{2}$-dependence of different higher twist spin-azimuthal asymmetries, which, apart from providing important information on quark-gluon correlations are needed for understanding of possible corrections from higher twists to leading twist observables.

Although, the Target Fragmentation Region (TFR) of DIS, when the hadrons are created from the target remnant, carries important information about the spin and flavor structure of the nucleon, it has not been studied systematically in experiments due to lack of theory fundamentals. One of the main physical questions in the TFR is how the diquark-like remnant system after the DIS process dresses itself up to become a full-fledged hadron, i.e., by which mechanism the quark-antiquark pairs restoring color neutrality are produced, and how this process is correlated with the spin of the target or/and the produced particles.

Recently the leading twist formalism for spin and transverse-momentum dependent fracture functions (FFs), describing conditional probabilities to produce a hadron of certain type in a target fragmentation for a given flavor of struck quark has been developed [18, 19]. The production of two hadrons in polarized SIDIS, with one spinless hadron produced in the current fragmentation region (CFR) and another in the TFR, would provide access to the full set of leading twist FFs [19]. In case of double hadron production process $l(\ell)+N(P) \rightarrow l\left(\ell^{\prime}\right)+h_{1}\left(P_{1}\right)+h_{2}\left(P_{2}\right)+X$, at leading order, the cross-section includes all fracture functions:

$$
\begin{gathered}
\frac{d \sigma^{l(\ell, \lambda)+N(P, S) \rightarrow l\left(\ell^{\prime}\right)+h_{1}\left(P_{1}\right)+h_{2}\left(P_{2}\right)+X}}{d x d y d z_{1} d \zeta_{2} d^{2} \overrightarrow{P_{T 1}} d^{2} \overrightarrow{P_{T 2}} d \phi_{S}}= \\
\frac{\alpha^{2} x_{B}}{Q^{4} y}\left[1+(1-y)^{2}\right]\left(\sigma_{U U}+S_{\|} \sigma_{U L}+S_{\perp} \sigma_{U T}+\lambda D_{l l} \sigma_{L U}+\lambda S_{\|} D_{l l} \sigma_{L L}+\lambda S_{\perp} D_{l l} \sigma_{L T}\right),
\end{gathered}
$$

where $D_{l l}(y)=y(2-y) / 1+(1-y)^{2}$. The subscripts in the structure functions $\sigma_{U T, U L, L T}$, specify the beam (first index) and target (second index) polarization ( $U, L, T$ for unpolarized, longitudinally 
and transversely polarized targets, and $U, L$ for unpolarized and longitudinally polarized beam).
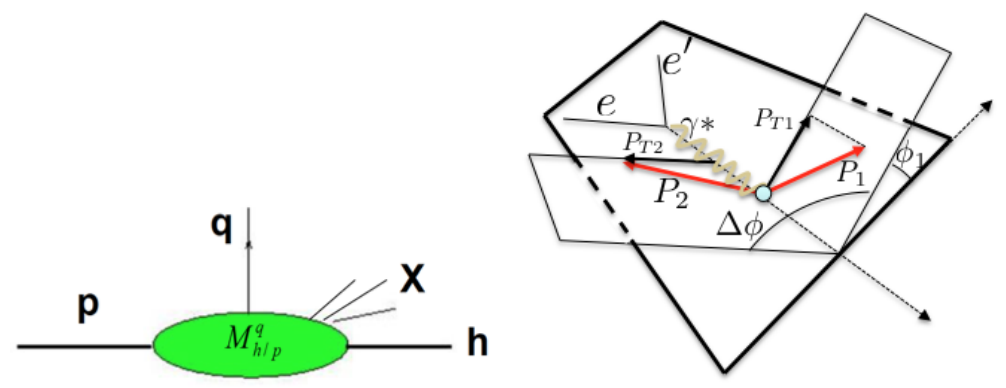

Figure 1: The fracture function defining the conditional probability to produce the hadron $\mathrm{h}$ when a quark $\mathrm{q}$ is struck in a proton target(left) and the kinematic plane for back-to-back (b2b) hadron production in SIDIS (right) .

For an unpolarized target expressions there are two contributions $\sigma_{U U}$ and $\sigma_{L U}$, involving convolutions of unpolarized, longitudinally and transversely polarized quark FFs (see Fig. 1) and fragmentation functions of unpolarized, $D_{1}$, and transversely polarized quarks, $H_{1}^{\perp}$, [18]:

$$
\begin{gathered}
\sigma_{U U}=F_{0}^{\hat{u} \cdot D_{1}}-D_{n n}\left[\frac{P_{T 1}^{2}}{m_{1} m_{N}} F_{k p 1}^{\hat{t}^{\perp} \cdot H_{1}^{\perp}} \cos \left(2 \phi_{1}\right)+\frac{P_{T 1} P_{T 2}}{m_{1} m_{2}} F_{p 1}^{\hat{t}^{h} \cdot H_{1}^{\perp}} \cos \left(\phi_{1}+\phi_{2}\right)\right. \\
\left.+\left(\frac{P_{T 2}^{2}}{m_{1} m_{N}} F_{k p 2}^{\hat{t}^{\perp} \cdot H_{1}^{\perp}}+\frac{P_{T 2}^{2}}{m_{1} m_{2}} F_{p 2}^{\hat{t}^{h} \cdot H_{1}^{\perp}}\right) \cos \left(2 \phi_{2}\right)\right] . \\
\sigma_{L U}=-\frac{P_{T 1} P_{T 2}}{m_{2} m_{N}} F_{k 1}^{\hat{l}^{\perp h} \cdot D_{1}} \sin \left(\phi_{1}-\phi_{2}\right),
\end{gathered}
$$

The structure functions $F_{\ldots} \ldots$ are specific convolutions [19] of fracture and fragmentation functions depending on $x, z_{1}, \zeta_{2}, P_{1 \perp}^{2}, P_{2 \perp}^{2}, \vec{P}_{1 \perp} \cdot \vec{P}_{2 \perp}$. The hadron 1 produced in the CFR $\left(x_{F 1}>0\right)$ is described by standard scaled variable $z_{1} \simeq P \cdot P_{1} / P \cdot q$, and its transverse momentum $\vec{P}_{T 1}$ (with magnitude $P_{T 1}$ and azimuthal angle $\left.\phi_{1}\right)$ and the hadron $2, h_{2}$ in the TFR $\left(x_{F 2}<0\right)$ is described by similar variables, $\zeta_{2} \simeq E_{2} / E$ and $\vec{P}_{T 2}\left(P_{T 2}\right.$ and $\left.\phi_{2}\right)$, where $x_{F}$ is Feynman variable defining the fraction of the longitudinal momentum of the hadron in the virtual photon-proton center of mass (CM) frame.

In electroproduction, the polarized lepton emits a virtual photon with non zero longitudinal polarization, which in turn selects preferentially one polarization state of the struck quark. With hadrons detected in the TFR, the beam SSAs appear already at leading order. With two hadrons detected in the final state, structure functions may depend also on the relative azimuthal angle of the two hadrons, generating a long range correlation between hadrons produced in CFR and TFR. The kinematic plane for back-to-back hadron production is shown on Fig. 1.

In the valence-quark region $(x>0.1)$ accessible at JLab with CLAS detector at $5.7 \mathrm{GeV}$ the polarization transfer from the beam is expected to be significant. High luminosity and high polarization of the electron beam makes CLAS an ideal place for studies of correlations between target and current fragmentation regions. The measurements of single spin asymmetry defined by Eq. 2 in semi-inclusive production of protons and charged pions in coincidence with the scattered electron in hard scattering kinematics $\left(Q^{2}>1 \mathrm{GeV}^{2}, W^{2}>4 \mathrm{GeV}^{2}\right)$ have been performed by the CLAS 
collaboration using $5.5 \mathrm{GeV}$ and $5.7 \mathrm{GeV}$ longitudinally polarized electron beams scattering off a 5-cm-long liquid-hydrogen target (CLAS e1f and e16 experiments).

Although, dihadron production in SIDIS requires higher energies and $Q^{2}$, than single hadron SIDIS, measurements of double-spin asymmetries at CLAS are already at $5.7 \mathrm{GeV}$ compatible with simple leading twist predictions for equality of double spin asymmetries in $e X, e \pi^{+} \pi^{-} X$, and $e \pi^{0} X$, assuming the sea quark contributions are negligible at large $\mathrm{x}_{\mathrm{B}}$ and fragmentation functions sum of charged pions are flavor independent. CLAS measurement of the double spin asymmetry from inclusive DIS (also from HERMES and SLAC) are consistent with CLAS measurements of double spin asymmetry in charged pion pair production. At low energies the multiplicities are low and one has to apply cuts on the missing mass of the final state to avoid contributions from exclusive states. Missing mass distributions for $e p \pi X$ events with final state protons detected in the backward region are shown in Fig. 2.
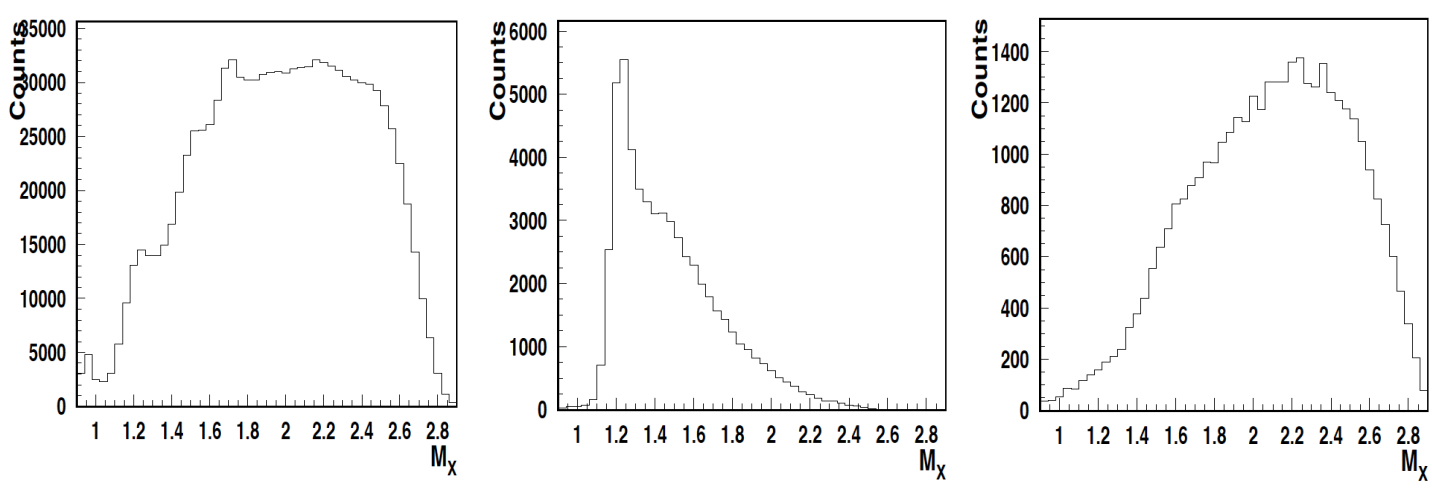

Figure 2: Missing mass (in GeV) distributions for $e^{\prime} p \pi^{+} X$ (left), $e^{\prime} p \pi^{-} X$ (center), and $e^{\prime} p \pi^{0} X$ (right) event sample at $5.7 \mathrm{GeV}$, with protons detected in the backward region.

Target and current fragmentation regions were selected by cuts on the $x_{F}$ variables of protons $\left(x_{F}<0\right)$ and pions $\left(x_{F}>0\right)$, in addition to standard data quality, vertex, acceptance, and fiducial cuts. Based on previous studies we have chosen $0.7>z>0.4$ as the canonical cut on the final state $\pi^{+}$to exclude contamination from exclusive events and decay pions from baryon resonances produced in the target fragmentation region. With a cut on the missing mass of the $e^{\prime} \pi^{+} X$ system, $M_{X}>1.4$ we have almost no data at $z>0.7$, but we use this cut as it was determined in Hall C that strong deviations for the quark-parton model occur at high $z$ [20]. The contamination from target fragmentation, higher twists, or other effects are important for $z<0.3$.

Choosing as independent azimuthal angles $\Delta \phi=\phi_{2}-\phi_{1}$ and $\phi_{2}$, the beam spin asymmetry could be defined as

$$
A_{L U}\left(x, z_{1}, \zeta_{2}, P_{T 1}^{2}, P_{T 2}^{2}, \Delta \phi\right)=\frac{\int \mathrm{d} \phi_{2} \sigma_{L U}}{\int \mathrm{d} \phi_{2} \sigma_{U U}}=\frac{-\frac{P_{T 1} P_{T 2}}{m_{2} m_{N}} F_{k 1}^{\hat{l}^{\perp h} \cdot D_{1}} \sin (\Delta \phi)}{F_{0}^{\hat{u} \cdot D_{1}}} .
$$

The beam spin asymmetry, $A_{L U}^{\sin \Delta \phi}$, has been calculated as a sinusoidal modulation of the difference of azimuthal angles of proton and $\pi^{+}$with respect to the lepton scattering plane, for different electron helicity states. The modulation was extracted for different bins in $x, z$ of the pion and the product of transverse momenta of final state proton and pion with respect to the virtual photon in the CM frame. 

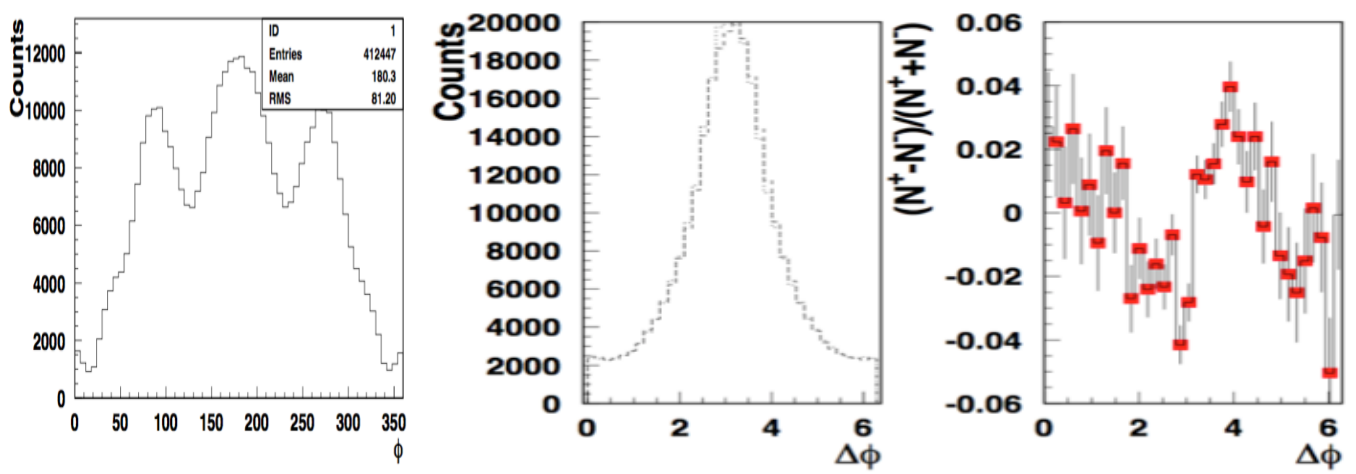

Figure 3: $\phi$-distributions of pions (left), the $\Delta \phi$, differences between proton and $\pi^{+}$azimuthal angles (middle) and difference in counts for positive and negative lepton helicities (right).

The azimuthal distributions of $\pi^{+}$and distribution over the $\Delta \phi$ for the full sample of events with detection of the scattered lepton, proton in the backward region and positive pion in the forward region are plotted in the Fig. 3. The difference in counts of positive and negative electron helicities indeed exhibit a sinusoidal behaviour. The missing mass dependence of the $A_{L U}$ for all three pions is shown in Fig. 4. Above the kinematic region where the target fragment may be an exclusive Delta $\left(M_{X}>1.5 \mathrm{GeV}\right)$ all three pions have similar behavior.
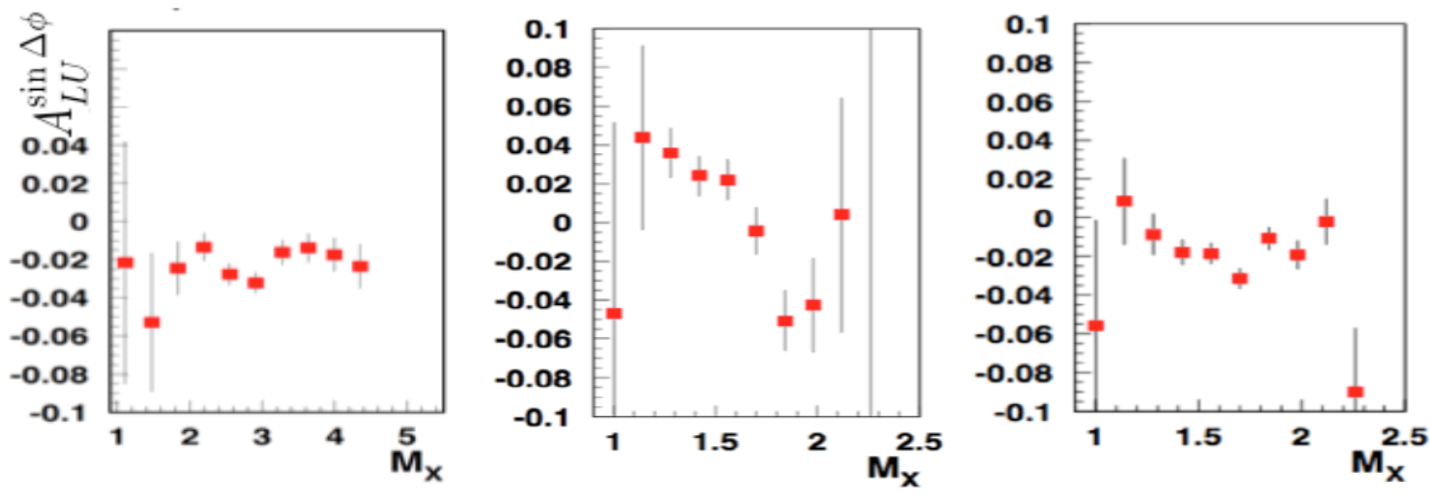

Figure 4: $A_{L U}$ dependence on missing mass of the $e \pi X$ system for $\pi^{+}$(left), $\pi^{-}$(middle) and $\pi^{0}$ (right).

The $P_{T}$-dependence of the SSA shows a trend for asymmetry to increase with increasing transverse momenta of pion and proton $P_{T 1}, P_{T 2}$, consistent with expectations from theory. The $x$-dependence of the the $A_{L U}^{\sin \phi}$ has been found to be consistent with asymmetry being large in the large- $\mathrm{x}_{\mathrm{B}}$ region, were the valence quark presence is very significant. For quantitative comparison with ongoing measurements as well as projections for future measurements of different $b 2 b$ processes using the CLAS12 and EIC one will need modeling of FFs, which can be modeled using different partonic models used to predict polarization of $\Lambda$ hyperons in the target fragmentation region of DIS, such as the meson cloud model [21] or intrinsic strangeness model for $\Lambda$ production in the target fragmentation region in deep-inelastic scattering [22, 23].

In conclusion, kinematic dependences of single spin asymmetries are measured in a wide kinematic range at CLAS with polarized beam and unpolarized hydrogen target. Significant single-spin 
asymmetries have been observed in back-to-back pion and proton electroproduction for the first time. A non-zero $A_{L U}$ in b2b SIDIS, measured for the first time, indicate that spin-orbit correlations between hadrons may be very significant opening a new avenue for studies of the complex nucleon structure in terms of quark and gluon degrees of freedom.

The JLab 12-GeV upgrade will provide the unique combination of wide kinematic coverage, high beam intensity (luminosity), high energy, high polarization, and advanced detection capabilities necessary to study the transverse momentum and spin correlations in di-hadron production in double-polarized semi-inclusive processes both in the target and current fragmentation regions. The large acceptance of the EIC combined with clear separation of target and current fragmentation regions would provide a qualitatively new tool to study the nucleon structure beyond the traditional current fragmentation.

We thank Aram Kotzinian for useful and stimulating discussions. This material is based upon work supported by the U.S. Department of Energy, Office of Science, Office of Nuclear Physics under contract DE-AC05-06OR23177.

\section{References}

[1] HERMES, A. Airapetian et al., Phys. Rev. Lett. 84 (2000) 4047, hep-ex/ 9910062.

[2] HERMES, A. Airapetian et al., Phys. Rev. D64 (2001) 097101, hep-ex/ 0104005.

[3] HERMES, A. Airapetian et al., Phys. Rev. Lett. 94 (2005) 012002, hep-ex/ 0408013.

[4] HERMES, A. Airapetian et al., Phys. Lett. B648 (2007) 164, hep-ex/ 0612059.

[5] COMPASS, V.Y. Alexakhin et al., Phys. Rev. Lett. 94 (2005) 202002, hep-ex/ 0503002.

[6] CLAS Collaboration, H. Avakian et al., Phys. Rev. D69 (2004) 112004, hep-ex/ 0301005.

[7] CLAS Collaboration, H. Avakian et al., AIP Conf. Proc. 792 (2005) 945, nucl-ex/ 0509032.

[8] H. Mkrtchyan et al., Phys. Lett. B665 (2008) 20, arXiv: hep-ph/ 0709 . 3020.

[9] CLAS, M. Osipenko et al., Phys. Rev. D80 (2009) 032004, arXiv: hep-ex/ 0809.1153.

[10] STAR, J. Adams et al., Phys. Rev. Lett. 92 (2004) 171801, hep-ex/ 0310058.

[11] PHENIX, M. Chiu, AIP Conf. Proc. 915 (2007) 539, nucl-ex/ 0701031.

[12] BRAHMS, I. Arsene et al., Phys. Rev. Lett. 101 (2008) 042001, arXiv:nucl-ex/0801.1078.

[13] Belle, K. Abe et al., Phys. Rev. Lett. 96 (2006) 232002, hep-ex/ 0507063.

[14] BELLE Collaboration, A. Vossen et al., Phys.Rev.Lett. (2011), arXiv:1104.2425.

[15] I. Garzia et al., Nuovo Cimento 33C (2010) 269.

[16] BaBar, I. Garzia, Nuovo Cim. C036 (2013) 181.

[17] H.H. Matevosyan et al., Phys. Rev. D92 (2015) 054028, arXiv:1502. 02669.

[18] M. Anselmino, V. Barone and A. Kotzinian, Phys. Lett. B699 (2011) 108, arXiv: 1102.4214.

[19] M. Anselmino, V. Barone and A. Kotzinian, Phys. Lett. B706 (2011) 46, arXiv:1109.1132.

[20] T. Navasardyan et al., Phys. Rev. Lett. 98 (2007) 022001, hep-ph / 0608214.

[21] W. Melnitchouk and A.W. Thomas, Z. Phys. A353 (1995) 311, arXiv: hep-ph/ 9508205.

[22] M.A. Alberg, J.R. Ellis and D. Kharzeev, Phys. Lett. B356 (1995) 113, arXiv: hep-ph/ 9503333.

[23] J.R. Ellis et al., Phys. Lett. B353 (1995) 319, arXiv: hep-ph/ 9412334. 